\title{
High frequency pressure oscillator for microcryocoolers
}

\author{
S. Vanapalli, H. J. M. ter Brake, H. V. Jansen, Y. Zhao, H. J. Holland, \\ J. F. Burger, and M. C. Elwenspoek \\ University of Twente, 7500 AE Enschede, The Netherlands
}

(Received 6 November 2007; accepted 16 March 2008; published online 10 April 2008)

\begin{abstract}
Microminiature pulse tube cryocoolers should operate at a frequency of an order higher than the conventional macro ones because the pulse tube cryocooler operating frequency scales inversely with the square of the pulse tube diameter. In this paper, the design and experiments of a high frequency pressure oscillator is presented with the aim to power a micropulse tube cryocooler operating between 300 and $80 \mathrm{~K}$, delivering a cooling power of $10 \mathrm{~mW}$. Piezoelectric actuators operate efficiently at high frequencies and have high power density making them good candidates as drivers for high frequency pressure oscillator. The pressure oscillator described in this work consists of a membrane driven by a piezoelectric actuator. A pressure ratio of about 1.11 was achieved with a filling pressure of $2.5 \mathrm{MPa}$ and compression volume of about $22.6 \mathrm{~mm}^{3}$ when operating the actuator with a peak-to-peak sinusoidal voltage of $100 \mathrm{~V}$ at a frequency of $1 \mathrm{kHz}$. The electrical power input was $2.73 \mathrm{~W}$. The high pressure ratio and low electrical input power at high frequencies would herald development of microminiature cryocoolers. (c) 2008 American Institute of Physics. [DOI: 10.1063/1.2906229]
\end{abstract}

\section{INTRODUCTION}

With emerging superconducting devices, ${ }^{1-3}$ infrared sensors for atmospheric studies, ${ }^{4,5}$ terahertz imaging sensors for military applications, ${ }^{6}$ and other electronic devices in microelectromechanical systems (MEMSs), the development of efficient and small cryocoolers to cool these devices is imperative. The existing cryocoolers are rather bulky and expensive. A cryocooler fabricated with microsystem technology is an ideal solution to integrate these devices directly on the cryocooler, enabling on-chip cryogenic devices. For achieving cryogenic temperatures below $100 \mathrm{~K}$, gas compression cycles are the viable options as it was shown that the lowest temperature ever achieved with optical coolers was about $220 \mathrm{~K}^{7}$ Multiple stages are required for thermoelectric coolers and these have very low performance below $200 \mathrm{~K}^{8}{ }^{8}$ Magnetic refrigerators operating from room temperature are rather inefficient. ${ }^{9}$ Among the gas cycles reviewed by Radebaugh, ${ }^{10}$ the Joule-Thomson cycle which is a recuperative type and the pulse tube cryocooler which is a regenerative type are of interest to miniaturization because of no moving parts in the cold end. Figure 1(a) shows the schematic of a closed cycle Joule-Thomson cycle. In this cycle, there is steady flow of refrigerant around the loop brought about by a reciprocating compressor with inlet and outlet valves. A pulse tube cryocooler [see Fig. 1(b)] operates with oscillating pressure and oscillating flow. A high pressure ratio of about 16:1 (Ref. 11) is required in the Joule-Thomson cycle where as pressure ratios of about 1.15-1.3 are common in pulse tube cryocoolers. ${ }^{12}$

The schematic of a pulse tube cryocooler is shown in Fig. 1(b). The inertance tube causes the flow to oscillate with the pressure in the right phase relationship. The pulse tube cryocooler requires adiabatic (no heat transfer between gas and the walls) compression and expansion in the pulse tube component to produce an oscillating temperature in the gas. As heat transfer between the gas and the pulse tube wall increases, the amplitude of the temperature oscillation decreases and the efficiency of the process decreases. For the process to be nearly adiabatic, the tube radius must be large compared to the thermal penetration depth $\delta_{t}$ in the helium gas. The thermal penetration depth varies with the frequency of operation $f$ as $\delta_{t} \propto f^{-1 / 2}{ }^{13}$ Conventional pulse tube cryocoolers operate at about $60 \mathrm{~Hz}$, leading to a lower size limit for efficient operation of such a cryocooler. Increasing the frequency to about $1 \mathrm{kHz}$, the tube radius of the pulse tube can be made considerably smaller, allowing the miniaturization of the cryocooler. Design rules for efficient high frequency operation of a regenerator was reported by Radebaugh and O'Gallagher, ${ }^{13}$ which, was experimentally verified on a macroscale by Vanapalli et al. ${ }^{14}$ The next step is to realize an efficient high frequency pressure oscillator to drive the microcold stage.

We have evaluated various mechanisms for actuating such a high frequency pressure oscillator: electrostatic, thermal, electromagnetic, and piezoelectric. Electrostatic actuators provide very low force and stroke and hence was eliminated from consideration. Thermal actuators have very low thermal-to-mechanical conversion efficiency and hence are not desirable. Electromagnetic actuation is widely used in commercial refrigerators but is also eliminated due to problems associated with cooling of the coil when scaled to smaller systems. Piezoelectric actuators have high power density, can operate at high frequencies, have good electrical-to-mechanical efficiency, and potentially have a very long operating life. However, they provide very low stroke, but this is not a severe drawback because of the low 


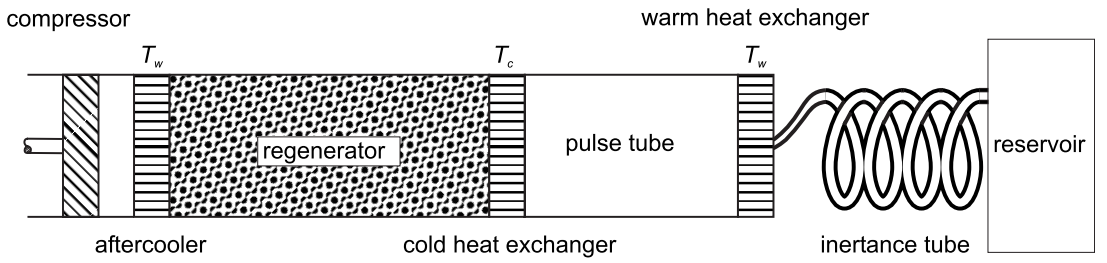

(b)

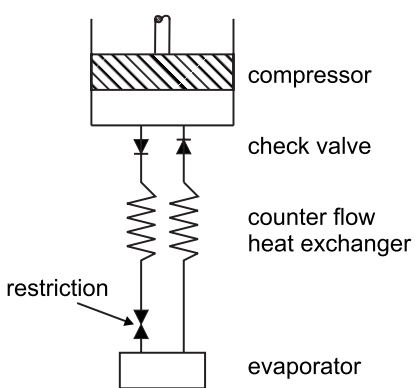

(a)

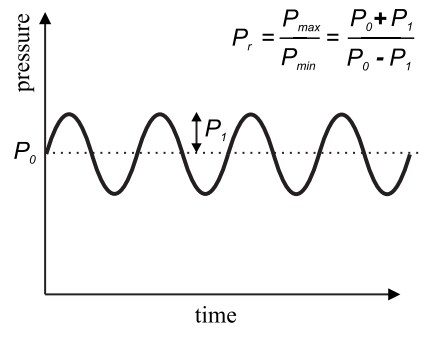

(c) dead volume in microcryocoolers. Because of excellent characteristics of piezoelectric actuators, this option was selected as a driver in the pressure oscillator.

The configuration of pressure oscillators are generally of two types: one is a piston moving in a cylinder and second is deflection of a membrane. A free-piston compressor of typical pulse tube cryocooler operates with a small diameter piston and a long stroke. ${ }^{15}$ The piston is suspended by springs combined with a clearance seal between the piston and the cylinder wall. The length is usually long compared to the diameter of the piston to reduce leakage looses. In contrast, membrane compressors have large diameter and a short stroke to limit the bending and tension stresses in the membrane. The membrane design was chosen for the pressure oscillator because it fits to the limited stroke capability of piezoelectric actuators.

\section{DESIGN}

Figure 1(c) shows the schematic of the trace of pressure in a pulse tube refrigerator. The sinusoidal pressure amplitude is represented by $P_{1}$ and the average pressure by $P_{0}$. The pressure ratio $P_{r}$, which is the ratio of the maximum to the minimum pressure over one cycle and is given by

$$
P_{r}=\frac{P_{0}+P_{1}}{P_{0}-P_{1}} .
$$

The gross refrigeration power $\dot{Q}_{r}$ of a pulse tube refrigerator is equal to the acoustic or PV power at the cold end of the regenerator and is given by

$$
\dot{Q}_{r}=\dot{W}_{\mathrm{PV}}=\frac{1}{2} P_{1} \dot{V}_{1, c} \cos \phi,
$$

where $\dot{V}_{1, c}$ is the amplitude of the sinusoidal volume flow rate at the cold end of the regenerator, and $\phi$ is the phase by which the volume flow leads the pressure. The volume flow rate $\dot{V}_{1, c}$ is related to the swept volume at the cold end $V_{1, c}$ as

$$
\dot{V}_{1, c}=j 2 \pi f V_{1, c},
$$

where $j$ is the imaginary unit and $f$ is the frequency of operation. The swept volume varies proportionally with temperature in the regenerator. The swept volume at the warm end of the regenerator $V_{1, w}$ is related to the swept volume at the cold end as

$$
\dot{V}_{1, w}=\frac{T_{w}}{T_{c}} \dot{V}_{1, c},
$$

where $T_{w}$ and $T_{c}$ are the temperatures at the warm and cold ends of the regenerator, respectively. The swept volume of the compressor should be larger than $V_{1, w}$ to account for dead-volume losses in the interconnects.

The pressure wave in the compression volume $V_{c}$ is created by the oscillating motion of the membrane. The generated pressure is a function of the temperature $T_{0}$ and the filling pressure $P_{0}$. The variation of temperature is rather small and can be neglected. In Fig. 2, the deflection profile of

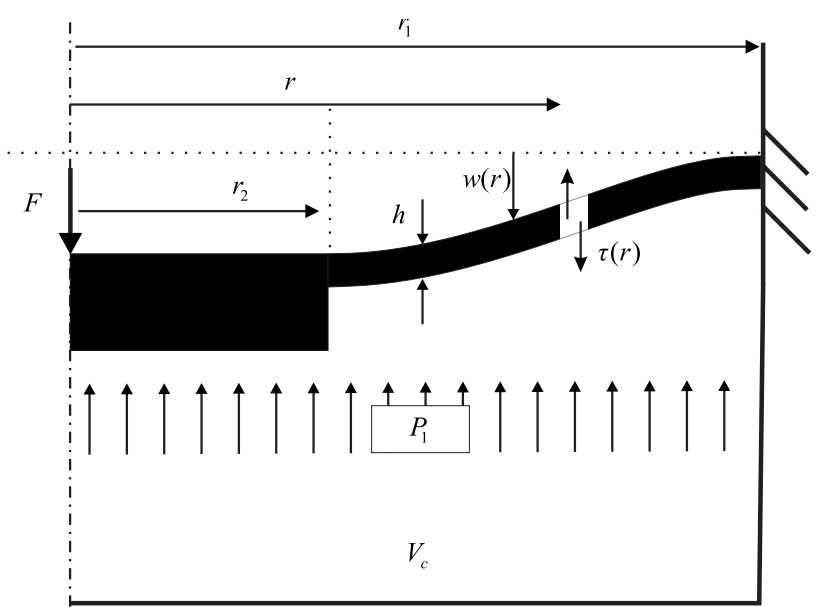

FIG. 2. Cross section of the deflection profile of the membrane subjected to a force by the actuator and the subsequent generation of differential pressure in the compression volume. 
the membrane under a load $F$ is shown; $w(r)$ is the deflection of the membrane at position $r$ and $\tau(r)$ is the shear force per unit length at position $r$. The center of the membrane has a rigid part of radius $r_{2}$, which is attached to the actuator. The membrane also has two laser drilled holes (not shown in Fig. 2) for static pressure equalization across the membrane. The dynamic pressure $P_{1}$ acting on the membrane gives a force balance

$$
2 \pi r \tau(r)=F-P_{1} \pi r^{2} .
$$

The differential equation for the deflection of the membrane is derived by using classical linear elasticity theory for plates $^{16}$

$$
\frac{d}{d r}\left[\frac{1}{r} \frac{d}{d r}\left(r \frac{d w(r)}{d r}\right)\right]=\frac{\tau(r)}{D},
$$

where $D$ is the flexural rigidity of the membrane given by

$$
D=\frac{E h^{3}}{12\left(1-\nu^{2}\right)} \text {. }
$$

In Eq. (7), $E$ is Young's modulus, $\nu$ is Poisson's ratio, and $h$ is the thickness of the membrane. The general solution of Eqs. (5) and (6) is

$$
\begin{aligned}
w(r)= & -\frac{r^{4}}{64 D} P_{1}+\left(+\frac{r^{2}}{8 \pi D} \ln (r)\right) F+C_{1}+\frac{1}{4} C_{2} r^{2} \\
& +C_{3} \ln (r) .
\end{aligned}
$$

The constants $C_{1}, C_{2}$, and $C_{3}$ are determined by applying the following boundary conditions:

$$
w\left(r_{1}\right)=0, \quad \frac{d w\left(r_{1}\right)}{d r}=0, \quad \frac{d w\left(r_{2}\right)}{d r}=0 .
$$

The volume of the gas $\Delta V$ displaced by the membrane is given by

$$
\Delta V=\pi r_{2}^{2} w(0)+\int_{r_{2}}^{r_{1}} 2 \pi r w(r) d r,
$$

which is equal to the volume change corresponding to the dynamic pressure $P_{1}$ that can be obtained from the ideal gas law for constant temperature conditions;

$$
\Delta V=-\frac{V_{c}}{P_{0}} P_{1},
$$

where $P_{0}$ is the average pressure in the compression volume.

The maximum force generated by the piezoactuator with stiffness $k_{p}$, on a load with stiffness $k_{l}$ is given by

$$
F=k_{p} \Delta L_{0}\left(\frac{k_{l}}{k_{p}+k_{l}}\right),
$$

where $\Delta L_{0}$ is the maximum no-load displacement of the actuator. The force $F$ generated by the piezoactuator depends on the no-load displacement $\Delta L_{0}$ at a certain frequency $f$ and voltage applied $V$ for a known load and piezostiffness. The load stiffness $k_{l}$ is given by

$$
k_{l}=k_{m}+k_{g}
$$

where $k_{m}$ is the membrane stiffness and $k_{g}$ is the stiffness of the gas in the compression volume $V_{c}$. Assuming isothermal
TABLE I. Specifications of the piezoelectric actuator and the dimensions of the membrane and the compression volume.

\begin{tabular}{cc}
\hline \hline Parameter & Value \\
\hline Stroke & $145 \mu \mathrm{m}$ \\
Stiffness & $1.0 \mathrm{~N} / \mu \mathrm{m}$ \\
Unloaded resonant frequency & $1400 \mathrm{~Hz}$ \\
Dimensions & $17 \times 28.5 \times 12 \mathrm{~mm}^{2}$ \\
Mass & $22 \mathrm{~g}$ \\
Membrane & \\
$r_{1}$ & $6.0 \mathrm{~mm}$ \\
$r_{2}$ & $3.0 \mathrm{~mm}$ \\
$h$ & $50 \mu \mathrm{m}$ \\
Compression space & \\
$V_{c}$ & $22.6 \mathrm{~mm}^{3}$ \\
\hline \hline
\end{tabular}

conditions in the compression volume $V_{c}$, the gas spring stiffness $k_{g}$ is given by ${ }^{17}$

$$
k_{g}=\frac{P_{0} A_{c}^{2}}{V_{c}},
$$

where $A_{c}$ is the membrane cross-sectional area.

In the present study, we aim at cooling a hightemperature superconducting device at $80 \mathrm{~K}$. Since such a device is nondissipating, a net cooling power of $10 \mathrm{~mW}$ is adequate. ${ }^{18}$ With the refrigerator operating at $f=1 \mathrm{kHz}, T_{w}$ $=300 \mathrm{~K}, T_{c}=80 \mathrm{~K}, \phi=-30^{\circ}, P_{r}=1.3, P_{0}=2.5 \mathrm{MPa}$, the $V_{1, w}$ is equal to $0.085 \mathrm{~mm}^{3}$ [Eqs. (2)-(4)], for a gross refrigeration power of $20 \mathrm{~mW}$ (assuming losses to be about $10 \mathrm{~mW}$ ). The compressed volume $V_{c}$ is equal to $22.6 \mathrm{~mm}^{3}$ and is larger than $V_{1, w}$ to simulate a load attached to the compressor. The dimensions of the membrane are given in Table I. The relationship between the force $F$ and dynamic pressure $P_{1}$ is derived from Eqs. (8) and (11) which, for the membrane dimensions given in Table $\mathrm{I}$, with an average pressure $P_{0}$ $=2.5 \mathrm{MPa}$ results in $P_{1}($ bar $)=0.11 F(N)$. The piezoactuator used in the experiments is shown in Fig. 3. The piezostack elongates in the axial direction and the stainless steel flexure attached to it amplifies the stroke into a perpendicular motion. The specifications of the actuator are given in Table I. The no-load displacement of the piezo was measured with a laser vibrometer with a resolution of $0.1 \mu \mathrm{m}$ which, works on the Mach-Zender interferometer principle. Figure 4 shows the amplitude of the displacement of the piezoactuator with voltage for several frequencies of operation. The dis-

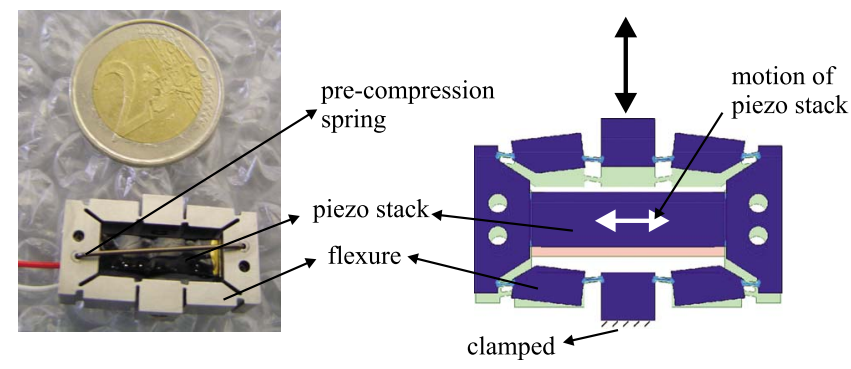

FIG. 3. (Color online) The piezoactuator used in the experiments. The motion of the piezostack is amplified by the flexure. A two Euro coin $(25.75 \mathrm{~mm})$ is shown for size comparison. 


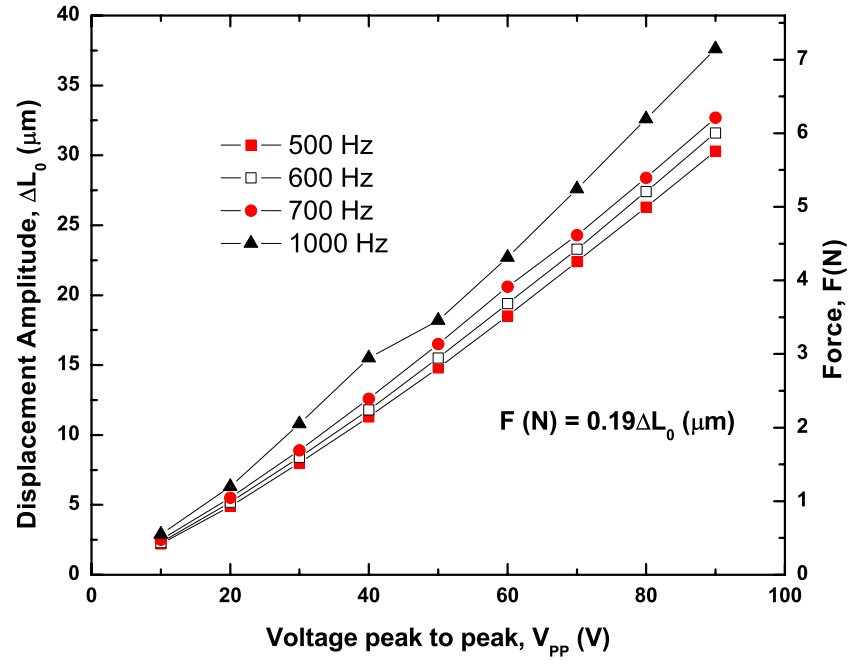

FIG. 4. (Color online) The no-load displacement amplitude of the piezoactuator with voltage for several frequencies of operation measured with a laser vibrometer.

placement varies rather linearly with voltage. For a peak-topeak voltage $V_{\mathrm{pp}}$ of $90 \mathrm{~V}$, the displacement was about $30 \mu \mathrm{m}$ at a frequency of $500 \mathrm{~Hz}$. With the same measuring technique, the no-load resonance frequency was determined as $1.43 \mathrm{kHz}$.

The layout drawing of the pressure oscillator is shown in Fig. 5. The piezoactuator was clamped to the cap of the oscillator. The membrane was laser cut from a stainless steel sheet of nominal thickness equal to $50 \mu \mathrm{m}$. Two holes of diameter less than about $10 \mu \mathrm{m}$ were laser drilled on the membrane for filling the compression chamber with high pressure gas. The holes were small enough to be leak tight under dynamic operation. A screw was laser welded on to the membrane. Fluidic and electrical feedthroughs were provided on the cap of the oscillator. The bottom flange clamps the membrane to the housing of the oscillator. A pressure tap on the flange was provided to measure the oscillating pressure generated in the compression volume $V_{c}$.

The stiffness of the membrane was obtained from Eq. (8) with $P_{1}=0$. The calculated stiffness of the membrane $k_{m}$ was about $0.145 \mathrm{~N} / \mu \mathrm{m}$. For an average pressure $P_{0}$ equal to
$2.5 \mathrm{MPa}$, the gas spring stiffness $K_{g}$ is about $0.088 \mathrm{~N} / \mu \mathrm{m}$. As a result, the load stiffness $k_{l}$ is equal to $0.233 \mathrm{~N} / \mu \mathrm{m}$.

\section{EXPERIMENTS}

The pressure oscillator was filled with nitrogen gas from a pressure regulated gas bottle. The pressure sensor located on the filling line close to the pressure oscillator measures the filling pressure in the system. A PT100 temperature sensor is glued on the piezostack to monitor the temperature of the piezostack. The current $i_{e}$ flowing into the piezostack was determined from the measured voltage across a calibrated resistor. From the traces of the voltage $E$ across the piezo and the current $i_{e}$, the electrical power $\dot{W}_{e}$ supplied to the piezo actuator is given by

$$
\dot{W}_{e}=\frac{1}{2}|E|\left|i_{e}\right| \cos \theta_{e},
$$

where $\theta_{e}$ is the phase angle between voltage and current. The dynamic pressure $P_{1}$ generated in the compression space of the oscillator was measured by a pressure sensor with a range of $0-3.5 \mathrm{MPa}$, sensitivity of $22.0 \mathrm{mV} / \mathrm{MPa}$ and a resolution of about 1 mbar. A lock-in amplifier was used to read the oscillating pressure.

Experiments were performed on the pressure oscillator with a filling pressure $P_{0}$ of $2.5 \mathrm{MPa}$ and varying the amplitude and frequency of the voltage input to the piezoactuator. Figure 6 shows the measured dynamic pressure amplitude $P_{1}$ for several frequencies of operation. The force was calculated from Eq. (12) with the no-load displacement $\Delta L_{0}$ obtained from Fig. 4 for an applied voltage on the piezoactuator. The calculated pressure with force as described in Sec. II is also plotted in the same figure. The measured pressure data agree rather good with the theoretical model.

Figure 7 shows the measured dynamic pressure amplitude $P_{1}$ as a function of the measured electrical power for several frequencies and a filling pressure $P_{0}$ of $2.5 \mathrm{MPa}$. For an applied peak-to-peak sinusoidal voltage of $100 \mathrm{~V}$, the measured pressure ratio $P_{r}$ at a frequency of $1 \mathrm{kHz}$ was about 1.11 and the measured electrical power input was about $2.73 \mathrm{~W}$. Figure 8 shows the calculated work done by the piezoactuator $P_{1} V_{1}$ as a function of measured electrical power for several frequencies and a filling pressure $P_{0}$ of

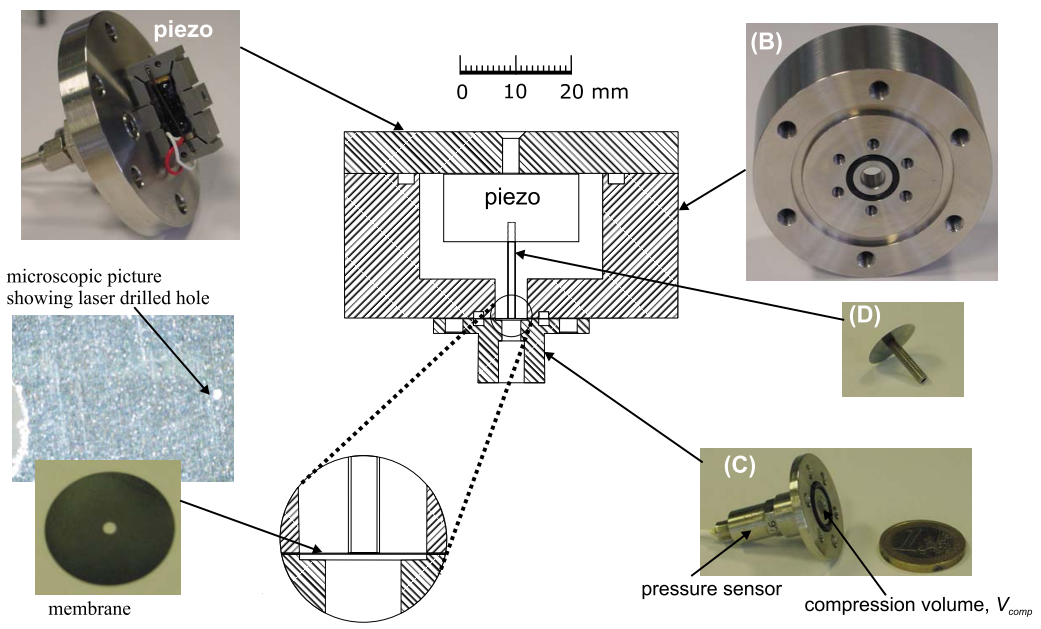

FIG. 5. (Color online) The layout of the pressure oscillator showing various components. The stainless steel membrane which was laser cut from a sheet is also shown. Two holes of diameter about $10 \mu \mathrm{m}$ were laser drilled, one of the hole is shown in the microscopic picture of the membrane. 


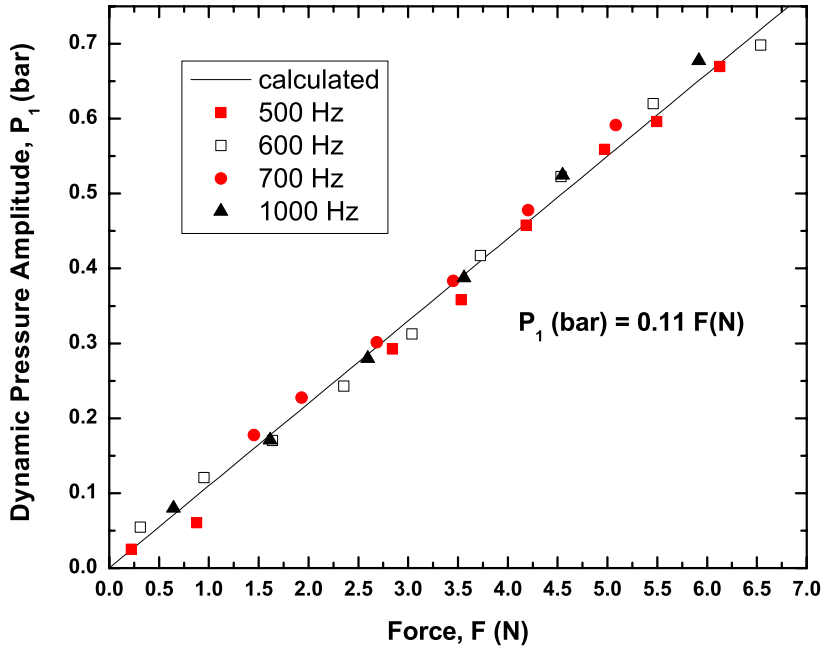

FIG. 6. (Color online) Calculated and experimentally generated oscillating pressure amplitude in the compression volume to the force exerted by the piezoactuator for several frequencies. The force is calculated from the voltage applied, the stiffness of the load and the no-load displacement in Fig. 4.

2.5 MPa. The PV energy $\left(P_{1} V_{1}\right)$, transferred at an operating frequency of $1 \mathrm{kHz}$ was about $0.17 \mathrm{~mJ}$ for an electrical input of $2.73 \mathrm{~W}$ when operating the piezoelectric actuator at $100 \mathrm{~V}$ peak-to-peak sinusoidal voltage. The PV power transmitted for the above conditions is about 1.07 W [using Eqs. (2) and (3) with $\phi=0$ ] which gives an efficiency of $20 \%$.

\section{DISCUSSION AND CONCLUSION}

A pressure oscillator operating at high frequency for driving micropulse cryocoolers was presented. Piezoelectric actuators produce large forces but low stroke and can operate over wide range of frequencies and hence are ideal drivers for low dead-volume MEMS cryocoolers. The theoretical model for deflection of membranes agrees well with experimental data. A pressure ratio of about 1.11 at a frequency of $1 \mathrm{kHz}$ was achieved when operating the piezo with a peakto-peak sinusoidal voltage of $100 \mathrm{~V}$. For these conditions, the work produced by the piezoactuator was about $0.17 \mathrm{~mJ}$

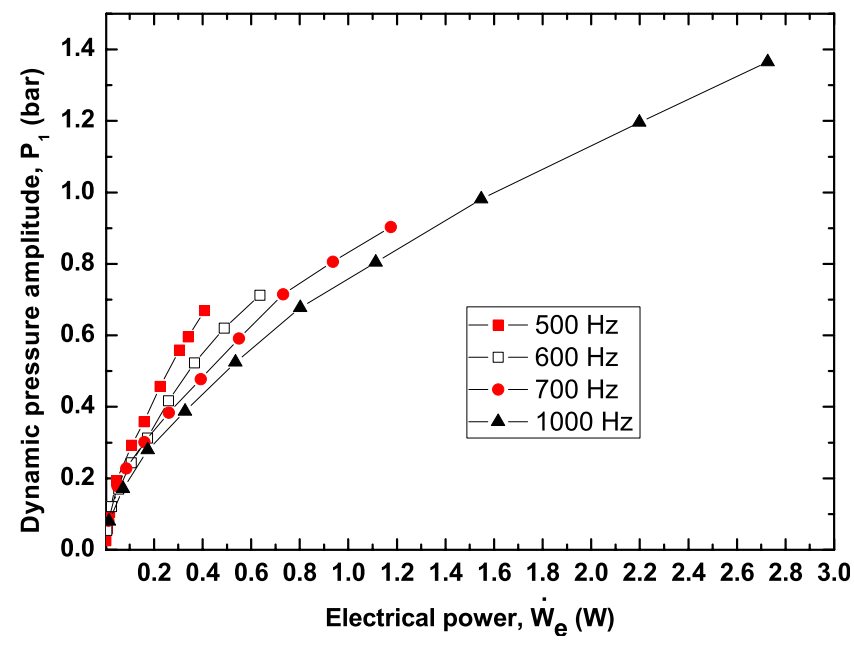

FIG. 7. (Color online) Measured oscillating pressure amplitude with electrical power supplied to the piezoactuator for several frequencies at a filling pressure of $2.5 \mathrm{MPa}$.

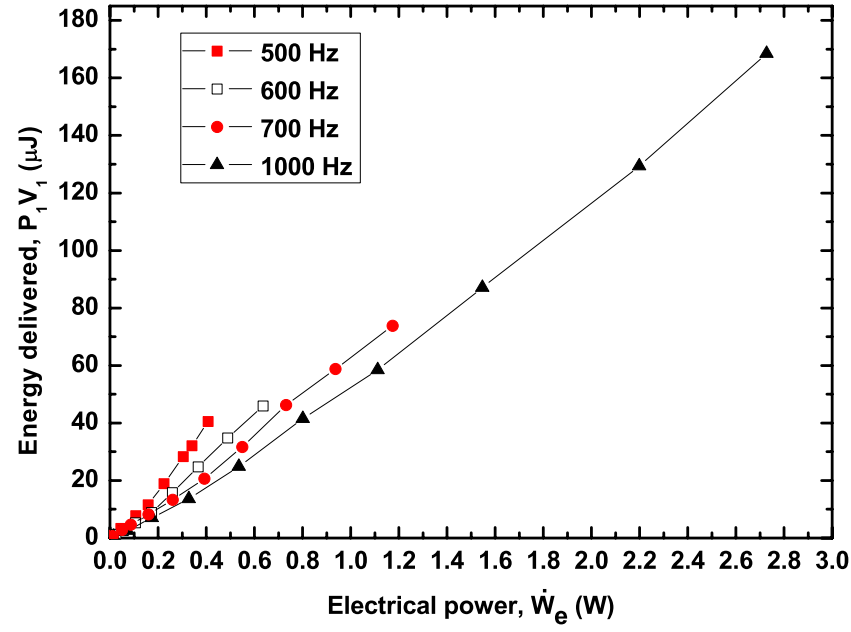

FIG. 8. (Color online) Work done by the piezoactuator with electrical power, for several frequencies at a filling pressure of $2.5 \mathrm{MPa}$.

for an electrical input power of $2.73 \mathrm{~W}$ which gives an efficiency of $20 \%$. Higher pressure ratios can be achieved by driving the actuator at higher voltages. This pressure oscillator can be directly coupled to a micropulse tube cold head resulting in the development of new generation of microcryocoolers. The development of high frequency pressure oscillators would pave the way to a new generation of on-chip micropulse tube cryocoolers.

Increasing the load stiffness $k_{l}$, by increasing the thickness of the membrane and decreasing the compression volume $V_{c}$ would result in large force exerted by the piezo. The large force, would provide high pressure ratio $P_{r}$, but low swept volume. Coupling the compression chamber with low void volume check valves as shown in Fig. 1(a), a steady high pressure flow can be generated. Operating the actuator at higher frequencies will provide sufficient high pressure flow to power a micro-Joule-Thomson cold tip. ${ }^{11}$

In a classical (ideal) Stirling refrigerator, the compression, expansion, and displacement of the gas is often represented by discontinuous piston and displacer motions. ${ }^{19}$ The fast response time of piezoelectric actuators would allow exploring nonsinusoidal operation of Stirling cycle which was not possible with conventional electromagnetic pressure oscillators. Further research must be carried out to utilize piezoelectric actuators to realize new variants of regenerative gas cycles.

\section{ACKNOWLEDGMENTS}

The authors acknowledge Klaas Smit for careful machining of the parts, Remco Sanders for many useful discussions on the experimental setup, and Marcel Dijkstra for introducing the laser vibrometer setup. This research was carried out within the STW (Dutch Technology Foundation) sponsored project "3LN Microcooler" (TTF.5677).

${ }^{1}$ C. H. Downey, Cryogenics 31, 48 (1991).

${ }^{2}$ A. E. Mahdi and D. J. Mapps, Sens. Actuators, A 81, 367 (2000).

${ }^{3}$ H. Nagata, J. Kobayashi, H. Matsuo, M. Akiba, and M. Fujiwara, Nucl. Instrum. Methods Phys. Res. A 559, 823 (2006).

${ }^{4}$ A. Rogalski, J. Appl. Phys. 93, 4355 (2003).

${ }^{5}$ M. Jhabvala, Sens. Actuators, A 42, 363 (2001). 
${ }^{6}$ P. H. Siegel, IEEE Trans. Microwave Theory Tech. 50, 910 (2002).

${ }^{7}$ T. R. Gosnell, Opt. Lett. 24, 1041 (1999).

${ }^{8}$ MELCOR Corp., 1040 Spruce Str., Trenton, NJ 08648, USA (http:// www.melcor.com).

${ }^{9}$ R. Radebaugh and G. Walker, Cryocoolers Part 2: Applications (Plenum, New York, 1983), pp. 129-175.

${ }^{10}$ R. Radebaugh, IEEE Proceedings on Electronic and Large Scale Applications of Superconductivity, 2004, Vol. 92, p. 1719.

${ }^{11}$ P. P. P. M. Lerou, G. C. F. Venhorst, C. F. Berends, T. T. Veenstra, M. Blom, J. F. Burger, H. J. M. ter Brake, and H. Rogalla, J. Micromech. Microeng. 16, 1919 (2006).

${ }^{12}$ R. Radebaugh, Pulse Tube Cryocoolers, edited by S. Kakac, H. Smirnov, and M. R. Avelino (Kluwer, Dordrecht, The Netherlands, 2003), pp. 415-434.

${ }^{13}$ R. Radebaugh and A. O'Gallagher, Adv. Cryog. Eng. 51, 1919 (2006).
${ }^{14}$ S. Vanapalli, M. Lewis, Z. Gan, and R. Radebaugh, Appl. Phys. Lett. 90, 072504 (2007).

${ }^{15}$ E. D. Marquardt, R. Radebaugh, and P. Kittel, Seventh International Cryocooler Conference Proceedings, Air Force Phillips Laboratory Report No. PL-CP-93-1001, 1993.

${ }^{16}$ S. P. Timoshenko and S. Woinowsky-Krieger, Theory of Plates and Shells (McGraw Hill, New York, 1959), Chap. 2.

${ }^{17}$ G. W. Swift, Thermoacoustics (American Institute of Physics, New York, 2002), pp. 61-63.

${ }^{18}$ P. P. P. M. Lerou, S. Vanapalli, H. V. Jansen, J. F. Burger, T. T. Veenstra, G. C. F. Venhorst, H. J. Holland, M. Elwenspoek, H. J. M. ter Brake, and H. Rogalla, Advances in Cryogenic Engineering: Transactions of the Cryogenic Engineering Conference CEC, 2006, Vol. 57, p. 977.

${ }^{19}$ G. Walker, Cryocoolers, Part 1: Fundamentals (Plenum, New York, 1983), Vol. 823, p. 977. 